Algebra univers. 50 (2003) 83-106

$0002-5240 / 03 / 010083-24$

DOI 10.1007/s00012-003-1822-4

(C) Birkhäuser Verlag, Basel, 2003

Algebra Universalis

\title{
Cancellative residuated lattices
}

\author{
P. Bahls, J. Cole, N. Galatos, P. Jipsen, and C. Tsinakis
}

\begin{abstract}
Cancellative residuated lattices are natural generalizations of lattice-ordered groups ( $\ell$-groups). Although cancellative monoids are defined by quasi-equations, the class $\mathcal{C}$ an $\mathcal{R} \mathcal{L}$ of cancellative residuated lattices is a variety. We prove that there are only two commutative subvarieties of $\mathcal{C} a n \mathcal{R} \mathcal{L}$ that cover the trivial variety, namely the varieties generated by the integers and the negative integers (with zero). We also construct examples showing that in contrast to $\ell$-groups, the lattice reducts of cancellative residuated lattices need not be distributive. In fact we prove that every lattice can be embedded in the lattice reduct of a cancellative residuated lattice. Moreover, we show that there exists an order-preserving injection of the lattice of all lattice varieties into the subvariety lattice of $\mathcal{C}$ an $\mathcal{R} \mathcal{L}$.

We define generalized MV-algebras and generalized BL-algebras and prove that the cancellative integral members of these varieties are precisely the negative cones of $\ell$-groups, hence the latter form a variety, denoted by $\mathcal{L G}^{-}$. Furthermore we prove that the map that sends a subvariety of $\ell$-groups to the corresponding class of negative cones is a lattice isomorphism from the lattice of subvarieties of $\mathcal{L G}$ to the lattice of subvarieties of $\mathcal{L G}^{-}$. Finally, we show how to translate equational bases between corresponding subvarieties, and briefly discuss these results in the context of R. McKenzie's characterization of categorically equivalent varieties.
\end{abstract}

\section{Introduction}

A residuated lattice-ordered monoid, or residuated lattice for short, is an algebra $\mathbf{L}=\langle L, \wedge, \vee, \cdot, e, \backslash, /\rangle$ such that $\langle L, \wedge, \vee\rangle$ is a lattice, $\langle L, \cdot, e\rangle$ is a monoid, and multiplication is both left and right residuated, with $\backslash$ and / as residuals. This means that for all $a, b, c \in L$,

$$
a \cdot b \leq c \quad \Leftrightarrow a \leq c / b \quad \Leftrightarrow \quad b \leq a \backslash c .
$$

M. Ward and R. P. Dilworth were the first to introduce the concept of a residuated lattice [26] as a generalization of ideal lattices of rings. In their original definition a residuated lattice was what we would call an integral commutative one. Basic properties and facts in this restricted setting were known, but in [7] one can find the first thorough study of residuated lattices in their generality. For a survey of residuated lattices we refer to [21].

Presented by W. Blok.

Received September 13, 2001; accepted in final form March 20, 2003.

2000 Mathematics Subject Classification: 06F05; 06D35, 06F15, $08 \mathrm{~B} 15$. 
The class $\mathcal{R} \mathcal{L}$ of all residuated lattices is easily seen to be a variety [7]. It includes algebras term equivalent to many well studied and diverse structures, such as generalized Boolean algebras, Brouwerian algebras, relative Stone algebras and $\ell$-groups. Thus, residuated lattices allow the study of all these algebras under a common language. For related work on subreducts of integral commutative residuated lattices we refer the reader to [6], [5] and [15].

In this paper we concentrate on cancellative residuated lattices, which form a variety by Lemma 2.5 . The variety of $\ell$-groups is the most studied cancellative variety of residuated lattices and it enjoys many interesting properties. We investigate whether some of the properties of $\ell$-groups extend to the whole variety of cancellative residuated lattices and construct examples that illustrate the limitations. In particular, we construct a cancellative commutative residuated lattice whose lattice reduct is not distributive and further prove that every lattice is a subreduct of a cancellative residuated lattice. Moreover, we provide sufficient conditions for a residuated lattice to have a distributive lattice reduct and prove that there are exactly two cancellative commutative atoms in the subvariety lattice, the varieties generated by $\mathbb{Z}$ and $\mathbb{Z}^{-}$.

C.-C. Chang introduced MV-algebras in 1958 as the algebraic counterpart of multi-valued propositional calculus. Even though MV-algebras are not cancellative, they are connected to abelian $\ell$-groups, as shown in [12], [23] (see also [13]). We introduce the variety of generalized MV-algebras and prove that the subvariety of cancellative members is precisely the class of negative cones of $\ell$-groups. As a result we obtain a finite equational basis for this class. We study further the connection between $\ell$-groups and their negative cones and show that their subvariety lattices are isomorphic. Finally, we describe an effective procedure of translating equational bases between corresponding subvarieties of these lattices.

\section{Preliminaries}

Throughout we use the convention that, in the absence of parentheses, $\cdot$ is performed first, followed by $\backslash, /$ and then $\vee, \wedge$.

The lattice of subvarieties of $\mathcal{R} \mathcal{L}$ is denoted by $\mathbf{L}(\mathcal{R} \mathcal{L})$. Some basic properties of subvarieties of $\mathcal{R} \mathcal{L}$ can be inferred from the fact that residuated lattices have lattice reducts. It follows for example that they are congruence distributive, and hence $\mathbf{L}(\mathcal{R} \mathcal{L})$ is distributive.

We quote below some results from [7] that we will be using in the remainder of the paper. The following lemma provides some useful identities that hold in all residuated lattices.

Lemma 2.1. [7] Let $\mathbf{L}$ be a residuated lattice. For all $a, b, c \in L$ we have: 
(i) $a(b \vee c)=a b \vee a c$ and $(b \vee c) a=b a \vee c a$.

(ii) $(a \wedge b) / c=(a / c) \wedge(b / c)$ and $c \backslash(a \wedge b)=(c \backslash a) \wedge(c \backslash b)$.

(iii) $a /(b \vee c)=(a / b) \wedge(a / c)$ and $(b \vee c) \backslash a=(b \backslash a) \wedge(c \backslash a)$.

(iv) $(a / c) c \leq a$ and $c(c \backslash a) \leq a$.

(v) $a(c / b) \leq a c / b$ and $(a \backslash c) b \leq a \backslash c b$.

(vi) $(c / b)(b / a) \leq c / a$ and $(a \backslash b)(b \backslash c) \leq a \backslash c$.

(vii) $c / b \leq(c / a) /(b / a)$ and $b \backslash c \leq(a \backslash b) \backslash(a \backslash c)$.

(viii) $b / a \leq(c / b) \backslash(c / a)$ and $a \backslash b \leq(a \backslash c) /(b \backslash c)$.

(ix) $c / b \leq c a / b a$ and $a \backslash c \leq b a \backslash b c$.

(x) $(c / a) / b=c / b a$ and $b \backslash(a \backslash c)=a b \backslash c$.

(xi) $a \backslash(c / b)=(a \backslash c) / b$.

(xii) $c \leq(a / c) \backslash a$ and $c \leq a /(c \backslash a)$.

(xiii) $a / e=a$ and $e \backslash a=a$.

(xiv) If $\mathbf{L}$ has a bottom element, 0 , then $\mathbf{L}$ also has a top element, 1, and for all $a \in L$ we have: $a 0=0 a=0, a / 0=0 \backslash a=1$ and $1 / a=a \backslash 1=1$.

For a residuated lattice $\mathbf{L}$, the negative part of $\mathbf{L}$ is $L^{-}=\{x \in L: x \leq e\}$. The negative cone of $\mathbf{L}$ is defined as $\mathbf{L}^{-}=\left\langle L^{-}, \wedge, \vee, \cdot, e, / \mathbf{L}^{-}, \backslash \mathbf{L}^{-}\right\rangle$, where

$$
a / \mathbf{L}^{-} b=a / b \wedge e \text { and } a \backslash^{\mathbf{L}^{-}} b=a \backslash b \wedge e .
$$

It is easy to check that $\mathbf{L}^{-}$is again a residuated lattice. For a class $\mathcal{K}$ of residuated lattices, $\mathcal{K}^{-}$denotes the class of negative cones of members of $\mathcal{K}$.

Given an element $a$ in a residuated lattice $\mathbf{L}$, define the left-conjugation map $\lambda_{a}$ and the right-conjugation map $\rho_{a}$ by $\lambda_{a}(x)=(a \backslash x a) \wedge e$ and $\rho_{a}(x)=(a x / a) \wedge e$, for all $x \in L$. A subset is normal if it is closed under $\lambda_{a}$ and $\rho_{a}$ for all $a \in L$. It is convex if for all $a \leq b$ in the subset, all elements between $a$ and $b$ are also in the subset.

The class $\mathcal{R} \mathcal{L}$ is an ideal variety, i.e., congruences are determined by their $e$ congruence classes, and these are further characterized as convex, normal subalgebras.

Theorem 2.2. [7] The lattice $\mathrm{CN}(\mathbf{L})$ of convex, normal subalgebras of a residuated lattice $\mathbf{L}$ is isomorphic to its congruence lattice $\operatorname{Con}(\mathbf{L})$. The isomorphism is given by the mutually inverse maps $\mathbf{H} \mapsto \theta_{\mathbf{H}}=\{\langle a, b\rangle:(a / b) \wedge e \in H$ and $(b / a) \wedge e \in H\}$ and $\theta \mapsto[e]_{\theta}$.

In fact the congruences are already determined by the negative part of $[e]_{\theta}$.

Theorem 2.3. [7] Let $\mathbf{S}$ be a convex, normal submonoid of $\mathbf{L}$ such that $S \subseteq L^{-}$. Then, defining the set $H_{S}$ by

$$
H_{S}=\{a: s \leq a \leq s \backslash e \text { for some } s \in S\},
$$


$\mathbf{H}_{S}$ is a convex, normal subalgebra of $\mathbf{L}$ and $S=H_{S}^{-}$. Conversely, if $\mathbf{H}$ is any convex, normal subalgebra of $\mathbf{L}$ then, setting $S_{H}=H^{-}, \mathbf{S}_{H}$ is a convex, normal submonoid of $\mathbf{L}$ and $H$ can be recovered from $S_{H}$ as described above. Moreover, the mutually inverse maps $\mathbf{H} \mapsto \mathbf{S}_{H}$ and $\mathbf{S} \mapsto \mathbf{H}_{S}$ establish a lattice isomorphism between the lattice $\mathrm{CN}(\mathbf{L})$ of convex, normal subalgebras of $\mathbf{L}$ and the lattice $\mathrm{CNM}(\mathbf{L})$ of convex, normal submonoids of $\mathbf{L}$ whose underlying sets are subsets of $L^{-}$.

Finally, the following lemma gives the construction of the convex, normal submonoid generated by a subset of the negative part.

Lemma 2.4. [7] Suppose $S \subseteq L^{-}$. Let

$$
\hat{S}=\left\{\left(\gamma_{1} \circ \cdots \circ \gamma_{n}\right)(s): n \in \omega, s \in S, \gamma_{i}=\rho_{a} \text { or } \gamma_{i}=\lambda_{a} \text { for some } a \in \mathbf{L}\right\}
$$

and let $P(\hat{S})$ be the set of all finite products of members of $\hat{S}$. Then the convex, normal submonoid generated by $S$ is $M(S)=\{x: a \leq x \leq$ e for some $a \in P(\hat{S})\}$.

A residuated lattice is said to be commutative if its monoid reduct is commutative and integral if it satisfies $x \wedge e \approx x$. The corresponding varieties will be denoted by $\mathcal{C R} \mathcal{L}$ and $\mathcal{I} \mathcal{R} \mathcal{L}$, respectively. For members of $\mathcal{C R} \mathcal{L}$, it is common to denote the residuals by $x \rightarrow y=x \backslash y=y / x$.

Similarly, a residuated lattice is said to be cancellative if its monoid reduct is cancellative. Although cancellativity is a quasi-equation in the variety of monoids, it is equivalent to an identity in residuated lattices.

Lemma 2.5. A residuated lattice is right cancellative as a monoid if and only if it satisfies the identity $x y / y \approx x$.

Proof. The identity $(x y / y) y=x y$ holds in any residuated lattice since $x y / y \leq x y / y$ implies $(x y / y) y \leq x y$, and $x y \leq x y$ implies $x \leq x y / y$, hence $x y \leq(x y / y) y$. By right cancellativity, we have $x y / y=x$. Conversely, suppose $x y / y=x$ holds, and consider elements $a, b, c$ such that $a c=b c$. Then $a=a c / c=b c / c=b$, so right cancellativity is satisfied.

Thus, a residuated lattice is cancellative if it satisfies both $x \backslash x y \approx y$ and $y x / x \approx$ $y$. The variety of cancellative residuated lattices will be denoted by $\mathcal{C}$ an $\mathcal{R} \mathcal{L}$.

An $\ell$-group is (term equivalent to) a residuated lattice that satisfies $x(x \backslash e)=e$. In this case it is common to write $x^{-1}=x \backslash e$. It follows quite easily that $x^{-1} x=e$, $x \backslash e=e / x, x / y=x y^{-1}$ and $x \backslash y=x^{-1} y$. Clearly the variety $\mathcal{L G}$ of all $\ell$-groups is a subvariety of $\mathcal{C}$ an $\mathcal{R} \mathcal{L}$. A standard reference on $\ell$-groups is [1].

The variety generated by all residuated chains is denoted by $\mathcal{R \mathcal { L } ^ { C }}$ and its commutative subvariety by $\mathcal{C} \mathcal{R} \mathcal{L}^{C}$. $\mathcal{R L}^{C}$ has been studied extensively in [7] while an equational basis for $\mathcal{C} \mathcal{R} \mathcal{L}^{C}$ is given by the two identities $e \wedge(x \vee y) \approx(e \wedge x) \vee(e \wedge y)$ and $e \leq x / y \vee y / x$ (see also [20]). 
The variety generated by $\mathcal{K}$ is denoted $\mathcal{V}(\mathcal{K})$, and $\mathcal{V}(\{\mathbf{L}\})$ is also abbreviated as $\mathcal{V}(\mathbf{L})$.

\section{Commutative cancellative varieties}

As usual, the abelian lattice-ordered group based on the integers is denoted by $\mathbb{Z}$. A well known result due to Weinberg [27] (see also [1]) asserts that $\mathcal{V}(\mathbb{Z})$ is the variety of abelian $\ell$-groups. We show in Corollary 7.2 that $\mathcal{V}\left(\mathbb{Z}^{-}\right)$consists of all negative cones of abelian $\ell$-groups.

Theorem 3.1. The only atom in $\mathbf{L}(\mathcal{R L})$ below the variety of cancellative, integral residuated lattices is $\mathcal{V}\left(\mathbb{Z}^{-}\right)$.

Proof. Let $\mathbf{L}$ be a nontrivial cancellative, integral residuated lattice. Consider an element $x \neq e$. Then by cancellativity, $x^{n}<x^{m}$ for all integers $n>m \geq 0$. The set $\left\{x^{n}: n \in \omega\right\}$ is closed under meets and joins, since it is a chain, and it is obviously closed under multiplication. We get from cancellativity that $x^{n} / x^{m}=$ $x^{n-m} x^{m} / x^{m}=x^{n-m}$ for $n>m \geq 0$. On the other hand, if $n \leq m$, then $x^{n} \geq e x^{m}$, so $x^{n} / x^{m}=e$, as $e$ is the largest element. Hence this set is a subalgebra, and it is clearly isomorphic to $\mathbb{Z}^{-}$. Therefore $\mathbb{Z}^{-}$is contained in every nontrivial variety of cancellative, integral residuated lattices.

The preceding result may be compared to an analogous result by W. Blok and J. Raftery in [6]. They show that the quasivariety of pocrims generated by the natural numbers is minimal in the lattice of quasivarieties of cancellative pocrims (pocrims are the division, multiplication and $e$ subreducts of integral commutative residuated lattices).

Lemma 3.2. The identities $x \backslash x \approx e \approx x / x$ hold in any cancellative residuated lattice.

Theorem 3.3. The only atoms below the variety of cancellative, commutative residuated lattices are $\mathcal{V}\left(\mathbb{Z}^{-}\right)$and $\mathcal{V}(\mathbb{Z})$.

Proof. Let $\mathbf{L}$ be a nontrivial cancellative, commutative residuated lattice. Since $x \leq e$ implies $e \leq e / x$, we have two cases:

Case 1. For all $x \in L^{-}, e / x=e$. Then $L^{-}=L$ since $y \leq e /(y \backslash e \wedge e)=e$ for all $y \in L$, so by the previous theorem it has a copy of $\mathbb{Z}^{-}$as a subalgebra. Therefore, $\mathbb{Z}^{-}$is contained in $\mathcal{V}(\mathbf{L})$.

Case 2. There is $a \in L^{-}$such that $e / a>e$. Then we consider an equivalence $\sim$ on $\mathbf{L}$ defined by $x \sim y$ iff $e / x=e / y$. Let $\theta$ be the least congruence containing $\sim$. First we observe that $\mathbf{L} / \theta$ is an $\ell$-group, since $e /(e / x) x=(e / x) /(e / x)=e$ by the preceding lemma, hence $(e / x) x \sim e$. Therefore $\mathbf{L} / \theta \models(e / x) x \approx e$, which 
is a defining identity of $\ell$-groups in the setting of residuated lattices. Next we need to show that $\mathbf{L} / \theta$ is not trivial. We will show that $a \notin[e]_{\theta}$. Assume not. $[e]_{\theta}$ is a convex, normal subalgebra of $\mathbf{L}$ generated by $K=\{x \in \mathbf{L}: x \sim e\}$ (by Theorem 2.2). As all of these generators are negative, and so is $a$, we can use Theorem 2.3 to conclude that $a$ is in the convex, normal submonoid of $\mathbf{L}$ generated by $K$. We can also delete "normal" from the previous statement, since conjugates of negative elements $y$ in $\mathbf{L}$ satisfy

$$
e \geq \rho_{x}(y)=x y / x \wedge e=y x / x \wedge e \geq y \wedge e=y .
$$

So using Lemma 2.4, we finally arrive at $b_{1} b_{2} \cdots b_{n} \leq a<e$ for some $b_{1}, \ldots, b_{n} \in K$. By Lemma 2.1(x),

$$
e / b_{1} b_{2} \cdots b_{n}=\left(e / b_{n}\right) / b_{1} b_{2} \cdots b_{n-1}=e / b_{1} b_{2} \cdots b_{n-1}=\cdots=e / b_{1}=e,
$$

so $e=e / b_{1} b_{2} \cdots b_{n} \geq e / a \geq e$, and hence $e / a=e$. But this contradicts our original assumption that $e / a>a$.

We proved that $\mathbf{L} / \theta$ is a nontrivial $\ell$-group, and every nontrivial subalgebra of it generated by a negative element is isomorphic to $\mathbb{Z}$. Therefore $\mathbb{Z}$ is contained in $\mathcal{V}(\mathbf{L})$.

N. Galatos has recently strengthened the preceding result in [17] by showing that $\mathcal{V}\left(\mathbb{Z}^{-}\right)$and $\mathcal{V}(\mathbb{Z})$ are the only cancellative atoms in $\mathbf{L}(\mathcal{R} \mathcal{L})$.

We now give an example of an integral, cancellative, commutative residuated chain that is not the negative cone of an $\ell$-group, even though its semigroup reduct is isomorphic to the semigroup reduct of the negative cone of an $\ell$-group.

Example 3.4. Let $\mathbf{F}$ be the universe of the free 2-generated commutative monoid on $a$ and $b$. We denote the empty word by $e$ and order $\mathbf{F}$ by dual shortlex order, i.e. for words $\alpha, \beta \in F$ we have $\alpha \leq \beta$ iff $|\alpha|>|\beta|$, or $|\alpha|=|\beta|$ and $\alpha<_{\text {lex }} \beta$ in the lexicographic order generated by $b<a$. For example,

$$
e>a>b>a^{2}>a b>b^{2}>a^{3}>a^{2} b>a b^{2}>b^{3}>\cdots
$$

Then $\mathbf{F}=\langle F, \wedge, \vee, \cdot, e, \backslash, /\rangle$ is a cancellative, commutative, integral residuated chain. However it is not the negative cone of an $\ell$-group. Indeed, it is easy to see that the negative cone of an $\ell$-group satisfies the law $(x / y) y \approx x \wedge y$ (see Corollary 6.3), whereas we have $(b / a) a=a^{2} \neq b=a \wedge b$ in $\mathbf{F}$.

\section{Lattice reducts of cancellative residuated lattices}

The following result generalizes a result in [2].

Proposition 4.1. The following conditions are equivalent in a lattice $\mathbf{L}$.

(i) $\mathbf{L}$ is distributive. 

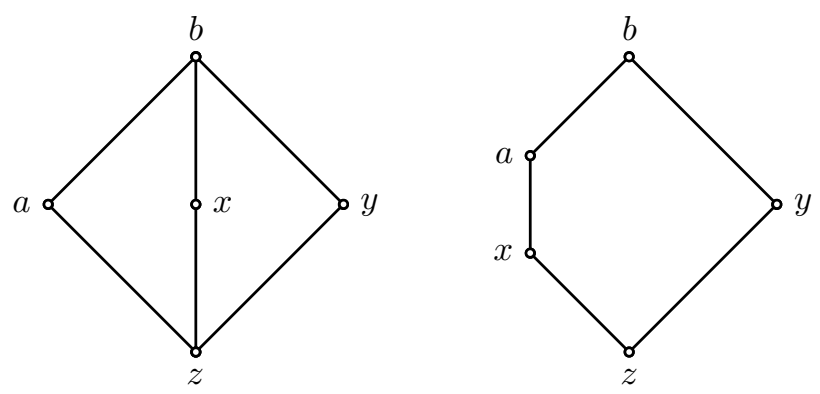

FiguRe 1

(ii) For all $a, b \in L$ with $a \leq b$, there exists a join-endomorphism $f$ of $\mathbf{L}$ such that $f(b)=a$ and $f(x) \leq x$, for all $x \in L$.

(iii) The dual of (ii): for all $a, b \in L$ with $a \leq b$, there exists a meet-endomorphism $f$ of $\mathbf{L}$ such that $f(a)=b$ and $x \leq f(x)$, for all $x \in L$.

Proof. (i) implies (ii), since we can take $f$ to be the map $f(x)=a \wedge x$. Conversely, suppose (ii) holds, and consider the lattices in Figure 1. If either one of them is a sublattice of $\mathbf{L}$, then we have $a=f(b)=f(x \vee y)=f(x) \vee f(y)$, hence $f(y) \leq a$. By assumption, $f(y) \leq y$, so $f(y) \leq a \wedge y=z$. But then $x \geq f(x) \vee f(y)=a$, which is a contradiction. Hence $L$ does not contain either of these lattices, and is therefore distributive. The equivalence of (i) and (iii) follows by duality.

We use the preceding result to establish three sufficient conditions for distributivity. That the second condition implies distributivity was first proved by Bosbach in [9].

Corollary 4.2. For residuated lattices, any of the following identities imply the distributive law:

(i) $x / x \approx e$ and $(x \vee y) / z \approx x / z \vee y / z$

(ii) $x(x \backslash(y \wedge e)) \approx x \wedge y$

(iii) $x \backslash x y \approx y, x y \approx y x$ and $x(y \wedge z) \approx x y \wedge x z$.

Proof. In each case one may use the preceding lemma with an appropriate definition of the endomorphism. For (i) take $f(x)=(x / b) a$, and for (ii) take $f(x)=$ $x(b \backslash(a \wedge e))$; then the second condition of the lemma is satisfied. For (iii) take $f(x)=a \backslash x b$, in which case the third condition of the lemma holds. 


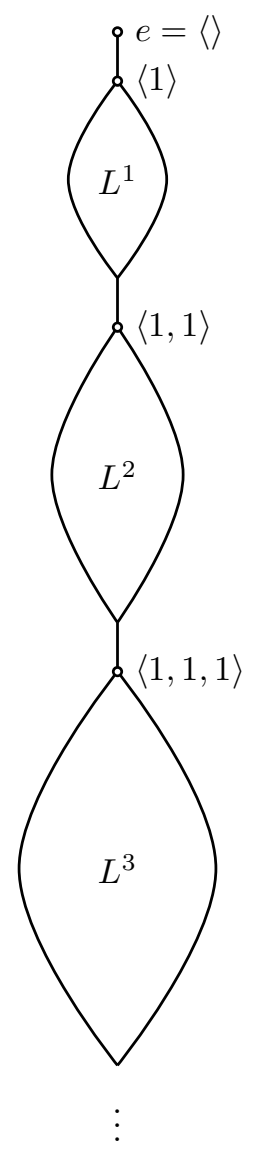

Figure 2. $\mathbf{L}^{*}$, a cancellative expansion of a given lattice $\mathbf{L}$

Note that (i) is a generalization of the well known fact that lattice reducts of $\ell$-groups are distributive. However, cancellativity alone is not enough to ensure distributivity, as the next result demonstrates.

Theorem 4.3. Any lattice is a sublattice of the lattice reduct of some simple, cancellative, integral residuated lattice.

Proof. Let $\mathbf{L}$ be a lattice. Since any lattice can be embedded in a lattice with a top element, we may assume that $\mathbf{L}$ has a top element, say 1 . Let $\mathbf{L}^{*}$ be the ordinal sum of $\mathbf{L}^{n}$ (the cartesian power, ordered pointwise) for $n=0,1,2, \ldots$, with $\mathbf{L}^{n}$ above $\mathbf{L}^{n+1}$ (see Figure 2), and define the multiplication by concatenation of sequences (where $L^{0}$ is the set containing the empty sequence). Then the monoid reduct of 
$\mathbf{L}^{*}$ is the free monoid generated by the elements in $L^{1}$, so $\mathbf{L}^{*}$ is cancellative. It is residuated since each block has a largest element, namely the constant sequence $\mathbf{1}_{k}=\langle 1,1, \ldots, 1\rangle \in L^{k}$. The left residual can be calculated explicitly:

$$
\begin{aligned}
& \left\langle x_{1}, \ldots, x_{m}\right\rangle \backslash\left\langle y_{1}, \ldots, y_{n}\right\rangle \\
& = \begin{cases}\left\langle y_{m+1}, \ldots, y_{n}\right\rangle & \text { if } m \leq n \text { and } x_{i} \leq y_{i} \text { for } 1 \leq i \leq m \\
\mathbf{1}_{k} & \text { otherwise, where } k=\max (n-m+1,0)\end{cases}
\end{aligned}
$$

and the right residual is similar. Note that the convex submonoid generated by $\langle 1\rangle$ is $L^{*}$, hence Theorem 2.2 implies that $\mathbf{L}^{*}$ is simple.

Note that the lattice reduct of $\mathbf{L}^{*}$ is in the variety generated by $\mathbf{L}$, since lattice varieties are closed under the operation of ordinal sum and adding a top element. If we take $\mathbf{L}$ to be $\mathbf{M}_{3}$, the 5-element modular lattice, then $\mathbf{L}^{*}$ is a 3 -generated modular, nondistributive, cancellative, integral residuated lattice. If we take $\mathbf{L}$ to be $\mathbf{N}_{5}$, the 5-element nonmodular lattice, then $\mathbf{L}^{*}$ is a nonmodular, cancellative, integral residuated lattice. In fact, given any lattice variety $\mathcal{V}$, we can define a subvariety of $\mathcal{C}$ an $\mathcal{R} \mathcal{L}$ by $\hat{\mathcal{V}}=\mathcal{V}\left(\left\{\mathbf{L}^{*}: \mathbf{L} \in \mathcal{V}\right\}\right)$.

Corollary 4.4. The map $\mathcal{V} \mapsto \hat{\mathcal{V}}$ is an order-preserving injection of the lattice of all lattice varieties into $\mathbf{L}(\mathcal{C}$ an $\mathcal{R} \mathcal{L})$.

The next example shows that there are even commutative, cancellative residuated lattices that are not distributive.

Example 4.5. Let $\mathbf{F}=\mathbf{F}_{\mathcal{C M}}(a, b, c)=\left\{a^{i} b^{j} c^{k}: i, j, k \in \omega\right\}$ be the 3-generated free commutative monoid. For a word $\alpha \in \mathbf{F}$, we denote the length of $\alpha$ by $|\alpha|$, and for $x \in\{a, b, c\}$, we define $|\alpha|_{x}$ to be the number of occurrences of $x$ in $\alpha$. The order on $\mathbf{F}$ is defined by $\alpha \leq \beta$ if $|\alpha|>|\beta|$, or $|\alpha|=|\beta|,|\alpha|_{b} \leq|\beta|_{b}$ and $|\alpha|_{c} \leq|\beta|_{c}$ (see Figure 3). Note that each block of words of the same length is a finite join-subsemilattice of a product of two chains, hence $\mathbf{F}$ is a lattice in which every join of elements is attained by a finite subjoin. Recall that a binary operation on a join-complete lattice is residuated iff it distributes over arbitrary joins. To see that the monoid operation of $\mathbf{F}$ is residuated, it therefore suffices to show that it distributes over finite joins. For two words $\alpha, \beta$ of the same length, we have $|\alpha \vee \beta|_{b}=\min \left(|\alpha|_{b},|\beta|_{b}\right)$, so

$$
\begin{aligned}
|(\alpha \vee \beta) \gamma|_{b} & =\min \left(|\alpha|_{b},|\beta|_{b}\right)+|\gamma|_{b} \\
& =\min \left(|\alpha|_{b}+|\gamma|_{b},|\beta|_{b}+|\gamma|_{b}\right)=|\alpha \gamma \vee \beta \gamma|_{b}
\end{aligned}
$$




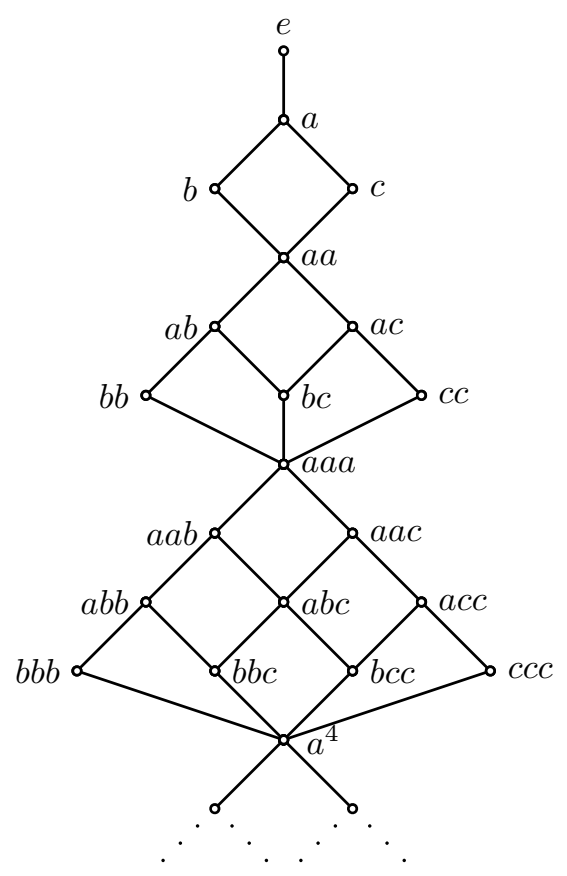

FIgURE 3. A nondistributive cancellative commutative example

and similarly for ||$_{c}$. It follows that $\mathbf{F}$ is a residuated lattice, and since the underlying commutative monoid is freely generated, $\mathbf{F}$ is cancellative. Finally, $\mathbf{F}$ is not distributive since $b b \vee(a b \wedge c c)=b b \vee(a a a)=b b$ while $(b b \vee a b) \wedge(b b \vee c c)=$ $(a b) \wedge(a a)=a b$.

It should be noted that the example above, while not distributive, is joinsemidistributive: if $\alpha \vee \beta=\alpha \vee \gamma=\delta$, then $\alpha \vee(\beta \wedge \gamma)=\delta$. One way to modify the example is to consider the free commutative monoid generated by $\{a, b, c, d\}$. The ordering on this new monoid is similar to the one above: $\alpha \leq \beta$ if $|\alpha|>|\beta|$, or $|\alpha|=|\beta|$ and $|\alpha|_{u} \leq|\beta|_{u}$, where $u \in\{b, c, d\}$. As before, one shows that this new ordering gives a residuated lattice which is not distributive. However, notice that now $b \vee c=b \vee d=a$ while $b \vee(c \wedge d)=b \vee(a a)=b$, so this new residuated lattice is not even join-semidistributive.

As noted in the introduction, the variety $\mathcal{C} \mathcal{R} \mathcal{L}^{C}$ generated by commutative residuated chains is axiomatized by $e \wedge(x \vee y) \approx(e \wedge x) \vee(e \wedge y)$ and $e \leq x / y \vee y / x$.

The second identity also holds in all $\ell$-groups (since they satisfy $e \leq x \vee x^{-1}$ ). Since $\mathcal{R} \mathcal{L}^{C}$ and $\mathcal{L G}$ both have distributive lattice reducts, the question arises 
whether this identity holds for all cancellative, distributive residuated lattices. However, if we take $\mathbf{L}=\{0,1\}$ to be the two-element chain, then the lattice $\mathbf{L}^{*}$ constructed above in Theorem 4.3 is a distributive, cancellative residuated lattice. To see that it fails $e \leq x / y \vee y / x$, take $x=\langle 0,1\rangle$ and $y=\langle 1,0\rangle$; then $x / y=\langle 1\rangle=y / x$.

A smaller generating class for $\mathcal{C} a n \mathcal{R} \mathcal{L}^{C}$. It is shown in [7] that the subdirectly irreducible algebras in $\mathcal{R} \mathcal{L}^{C}$ are precisely the residuated chains. So a generating set for $\mathcal{C}$ an $\mathcal{R} \mathcal{L}^{C}$ is the class of all cancellative residuated chains. The next result shows that it suffices to consider the subclass whose monoid reducts are countably generated free monoids.

Proposition 4.6. Every cancellative residuated chain is the homomorphic image of a residuated chain whose monoid reduct is a free monoid.

Proof. Let $\mathbf{C}$ be a cancellative residuated chain and let $C^{*}=\bigcup_{n \in \omega} C^{n}$, the free monoid generated by $C^{1}$, with the multiplication given by concatenation. The identity element of $\mathbf{C}$ is denoted by $e$ and the identity element of $\mathbf{C}^{*}$ is the empty tuple \langle\rangle . Note that these are not the same element. Let $\preceq$ be a dual shortlex order on $C^{*}$ generated by some dual well-ordering of $C$. This means $\alpha \preceq \beta$ iff $|\alpha|>|\beta|$, or $|\alpha|=|\beta|$ and $\alpha$ is lexicographically greater or equal to $\beta$ with respect to the chosen dual well-ordering of $C$. Finally, let $f: \mathbf{C}^{*} \rightarrow \mathbf{C}$ be the canonical monoid homomorphism, i.e. $f\left(\left\langle a_{1}, a_{2}, \ldots, a_{n}\right\rangle\right)=a_{1} a_{2} \cdots a_{n}$, where $a_{i} \in C$.

We now define a linear order on $\mathbf{C}^{*}$, and then prove that $\mathbf{C}^{*}$ is a residuated chain and $f$ is a residuated lattice homomorphism. For $\alpha, \beta \in C^{*}$ define $\alpha \leq \beta$ iff $f(\alpha)<f(\beta)$ in $\mathbf{C}$, or $f(\alpha)=f(\beta)$ and $\alpha \preceq \beta$. It follows from the definition that multiplication is order preserving, and hence join preserving.

Let $\alpha, \beta \in C^{*}$. Notice that if $\gamma \beta \leq \alpha$, then $f(\gamma \beta)=f(\gamma) f(\beta) \leq f(\alpha)$. So, we have that $f(\gamma) \leq f(\alpha) / f(\beta)$. Hence, if $\alpha / \beta$ exists in $\mathbf{C}^{*}$, then $f(\alpha / \beta) \leq f(\alpha) / f(\beta)$. (Similarly, we have that $f(\beta \backslash \alpha) \leq f(\beta) \backslash f(\alpha)$, if $\beta \backslash \alpha$ exists in $\mathbf{C}^{*}$.) Consider $\Gamma=\{\gamma: f(\gamma)=f(\alpha) / f(\beta)\}$.

Let $n=\max \{|\alpha|-|\beta|, 0\}$. Let $\gamma=\langle f(\alpha) / f(\beta), e, \ldots, e\rangle \in C^{n+1}$, which is in $\Gamma$. Now, $f(\gamma \beta)=(f(\alpha) / f(\beta)) f(\beta) \leq f(\alpha)$ and $|\gamma \beta|=1+n+|\beta|>|\alpha|$. Hence $\gamma \beta \leq \alpha$. Let $\Gamma^{\prime}=\{\gamma: \gamma \in \Gamma, \gamma \beta \leq \alpha\}$. In light of the preceding observations, we have that $\Gamma^{\prime} \neq \emptyset$. Also note that $\Gamma$ is dually well-ordered, and in particular $\delta=\bigvee \Gamma^{\prime}$ exists. Now

$$
\delta \beta=\bigvee_{\gamma \in \Gamma^{\prime}} \gamma \beta \leq \bigvee_{\gamma \in \Gamma^{\prime}} \alpha=\alpha .
$$

Therefore, if $\gamma \leq \delta$, then $\gamma \beta \leq \alpha$. If follows that $\gamma \beta \leq \alpha$ implies that $f(\gamma) \leq$ $f(\alpha) / f(\beta)$, which implies $\gamma \leq \delta$. Hence, $\delta=\alpha / \beta$ in $\mathbf{C}^{*}$. In a similar manner $\beta \backslash \alpha=\vee\{\gamma: f(\gamma)=f(\beta) \backslash f(\alpha), \beta \gamma \leq \alpha\}$. 
Notice that the paragraph above shows that $\alpha / \beta=\bigvee \Gamma^{\prime}$. So, we have that $f(\alpha / \beta) \geq f(\alpha) / f(\beta)$. Thus, we can conclude that $f(\alpha / \beta)=f(\alpha) / f(\beta)$. Similarly, we have that $f(\beta \backslash \alpha)=f(\beta) \backslash f(\alpha)$. Since $f$ is a monoid homomorphism and an order homomorphism by definition, we have that $f$ is a residuated lattice homomorphism. Thus $\mathbf{C}$ is a homomorphic image of $\mathbf{C}^{*}$.

Since the collection of all countable cancellative residuated chains generates $\mathcal{C}$ an $\mathcal{R} \mathcal{L}^{C}$, we have the following corollary.

Corollary 4.7. The collection of all residuated total orders on a countably generated free monoid is a generating set for the variety $\mathcal{C}$ an $\mathcal{R} \mathcal{L}^{C}$.

\section{Generalized MV-algebras and BL-algebras}

A residuated 0,1-lattice is a bounded residuated lattice with the additional constant operations 0 and 1 denoting the bottom and top element of the lattice. Since $1=0 / 0$ holds in any such lattice, it suffices to only include 0 in the similarity type. (Note that the top element, 1, of a residuated lattice, whenever it exists, does not have to coincide with the multiplicative identity $e$.)

MV-algebras were introduced as algebraic counterparts of the multi-valued logic of Eukasiewicz (see [13]). In this section we give a (somewhat nonstandard but term-equivalent) definition of MV-algebras as a subvariety of 0,1-residuated lattices. In our context, commutativity, integrality and the existence of a bottom element are not essential ingredients of the algebraic theory. This leads us to the definition of generalized MV-algebras as a subvariety of $\mathcal{R} \mathcal{L}$. Basic logic is another nonclassical logic that has recently received some attention [19]. It is included in multi-valued logic, and the algebraic counterparts are called BL-algebras. Here we also define them in the context of 0,1-residuated lattices, as well as the larger class of generalized BL-algebras. In the following section we prove that in the presence of cancellativity, generalized BL-algebras are generalized MV-algebras, and are precisely the negative cones of $\ell$-groups.

An $M V$-algebra is a commutative residuated 0,1-lattice that satisfies the identity $x \vee y \approx(x \rightarrow y) \rightarrow y$. This identity implies that the residuated lattice is also integral (see Lemma 5.2 below), hence we write 1 for the monoid identity and obtain algebras of the form $\mathbf{M}=\langle M, \wedge, \vee, \cdot, 1, \rightarrow, 0\rangle$.

Furthermore, one can show that $x y \approx(x \rightarrow(y \rightarrow 0)) \rightarrow 0, x \wedge y \approx x(x \rightarrow y)$ and of course $1 \approx 0 \rightarrow 0$, so it suffices to have $\rightarrow$ and 0 as fundamental operations (see e.g. [19], [24]). Such algebras are also known as Wajsberg algebras. In the original definition of MV-algebras, Chang [11] uses the operations $+, \cdot, \neg, 0,1$, where $\neg x=x \rightarrow 0$ and $x+y=\neg(\neg x \cdot \neg y)$. In this case $\rightarrow$ is definable by $x \rightarrow y \approx \neg x+y$, 
and $\langle M,+, \cdot, \neg, 0,1\rangle$ is an MV-algebra if $\langle M, \cdot, 1\rangle$ is a commutative monoid and satisfies

$$
\begin{gathered}
\neg \neg x \approx x, \quad 0=\neg 1, \quad x 0=0, \quad x+y=\neg(\neg x \cdot \neg y) \\
\text { and }(x \rightarrow y) \rightarrow y \approx(y \rightarrow x) \rightarrow x .
\end{gathered}
$$

Standard examples of MV-algebras are Boolean algebras, and the [0,1]-algebra defined on the unit interval, with $x \cdot y=\max (0, x+y-1)$ and $\neg x=1-x$ (in which case $x+y=\min (1, x+y)$ and $x \rightarrow y=\min (1,1-x+y))$.

The latter example can be generalized to abelian $\ell$-groups as follows. If $\mathbf{G}=$ $\langle G, \wedge, \vee, \cdot, \backslash, /, e\rangle$ is an abelian $\ell$-group and $a$ a positive element, then $\Gamma(\mathbf{G}, a)=$ $\langle[e, a], \wedge, \vee, \circ, a, \rightarrow, e\rangle$ is an MV-algebra, where $x \circ y=x y / a \vee e, x \rightarrow y=y a / x \wedge a$. Chang [12] proved that if $\mathbf{M}$ is a totally ordered MV-algebra then there is an abelian $\ell$-group $\mathbf{G}$ and a positive element $a$ of it such that $\mathbf{M} \cong \Gamma(\mathbf{G}, a)$. Moreover, Mundici [23] generalized the result to all MV algebras and proved that $\Gamma$ is an equivalence between the category of MV-algebras and the category of abelian $\ell$-groups with strong unit. A good reference for MV-algebras is [13]. See [18] for a generalization of this result to arbitrary $\ell$-groups.

A generalized $M V$-algebra (or GMV-algebra for short) is a residuated lattice that satisfies $x /((x \vee y) \backslash x) \approx x \vee y \approx(x /(x \vee y)) \backslash x$.

Note that MV-algebras are generalized in two directions: the existence of lattice bounds is not stipulated and the commutativity assumption is dropped. In particular, bounded commutative generalized MV-algebras are reducts of MV-algebras.

A $B L$-algebra is a commutative residuated 0,1-lattice that satisfies the identities $x \wedge y \approx x(x \rightarrow y)$ and $x \rightarrow y \vee y \rightarrow x=e$. Taking $x=e$ in the first identity, we have $e \wedge y=y$. Hence as for MV-algebras, BL-algebras are integral, and we may replace $e$ by 1 .

A generalized $B L$-algebra (or GBL-algebra for short) is a residuated lattice that satisfies $((x \wedge y) / y) y \approx x \wedge y \approx y(y \backslash(x \wedge y))$. The reader is referred to [18] for a detailed study of GMV-algebras and GBL-algebras. Note that GBL-algebras have distributive lattice reducts, by Corollary 4.2(ii) and Lemma 5.3(i).

We note that the GBL and GMV identities are equivalent to simpler quasiidentities.

\section{Lemma 5.1.}

(i) The GBL identity $y(y \backslash(x \wedge y)) \approx x \wedge y$ is equivalent to the quasi-identity $x \leq y \Rightarrow x=y(y \backslash x)$.

(ii) The $G M V$ identity $x /((x \vee y) \backslash x) \approx x \vee y$ is equivalent to the quasi-identity $x \leq y \Rightarrow y=x /(y \backslash x)$.

The next result shows that $\mathcal{G M V}$ is a subvariety of $\mathcal{G B} \mathcal{L}$. In particular, it follows that GMV-algebras are distributive. 
Lemma 5.2. Every GMV-algebra is a GBL-algebra.

Proof. We make use of the quasi-equational formulation from the preceding lemma. Assume that $x \leq y$ and let $z=y(y \backslash x)$. Note that $z \leq x$ and $y \backslash z \leq x \backslash z$. Hence,

$$
\begin{aligned}
x \backslash z & =((y \backslash z) /(x \backslash z)) \backslash(y \backslash z) & & \\
& =(y \backslash(z /(x \backslash z))) \backslash(y \backslash z) & & \text { since }(u \backslash v) / w=u \backslash(v / w) \\
& =(y \backslash x) \backslash(y \backslash z) & & \text { since } z \leq x \Rightarrow x=z /(x \backslash z) \\
& =(y(y \backslash x) \backslash z & & \text { since } u \backslash(v \backslash w)=v u \backslash w \\
& =z \backslash z . & &
\end{aligned}
$$

Therefore, $x=z /(x \backslash z)=z /(z \backslash z)=z$, as required. The proof of $x=(x / y) y$ is similar.

The following lemma provides an alternative equivalent axiomatization for GBL and GMV-algebras.

Lemma 5.3. (i) The GBL identity $y(y \backslash(x \wedge y)) \approx x \wedge y$ is equivalent to the identity $y(y \backslash x \wedge e)) \approx x \wedge y$.

(ii) The GMV identity $x /((x \vee y) \backslash x) \approx x \vee y$ is equivalent to the identity $x /(y \backslash x \wedge e)) \approx x \vee y$.

Proof. The proof will be immediate once we prove that each of the identities in the statement of the lemma imply the identity $x \backslash x \approx e$. To this end, note that in every residuated lattice,

$$
x \vee y \leq x /((x \vee y) \backslash x) \leq x /(y \backslash x \wedge e),
$$

for all elements $x, y$. Thus the identity $x /(y \backslash x \wedge e)) \approx x \vee y$ implies the GMV identity. Moreover, both the identities in (i) imply the quasi-identity

$$
e \leq y \Rightarrow e=y(y \backslash e)
$$

which states that every positive element has a right inverse. Next note that every element $x \backslash x$ is both positive and idempotent in any residuated lattice. Thus, in light of Lemma 5.2, each of the four identities in the statement of the lemma imply the identity $x \backslash x \approx e$, as was to be shown.

We will concentrate on the classes of integral GMV-algebras and integral GBLalgebras. Using the previous lemma, we can see that these varieties are axiomatized, relative to the variety of residuated lattices, by the identities $x /(y \backslash x) \approx x \vee y \approx$ $(x / y) \backslash x$ and $(x / y) y \approx x \wedge y \approx y(y \backslash x)$, respectively. 


\section{Characterizing cancellative, integral, generalized MV-algebras}

In this section we consider the relationship between a given class of residuated lattices and the corresponding class of negative cones. We prove below that there is a lattice isomorphism between the subvarieties of $\mathcal{L G}$ and the subvarieties of $\mathcal{L G}^{-}$. We also show how identities can be translated back and forth between two corresponding subvarieties.

We begin by recalling a standard construction for embedding certain cancellative monoids into groups (see, for example, [16]).

Lemma 6.1. Let $\mathbf{H}=\langle H, \cdot, e\rangle$ be a cancellative monoid such that aH $=H$ a for all $a \in H$. Then there is a group $Q(\mathbf{H})$ and an embedding $q: \mathbf{H} \rightarrow Q(\mathbf{H})$ such that every element of $Q(\mathbf{H})$ is of the form $q(a) q(b)^{-1}$ for some $a, b \in H$.

Proof. From the assumption that $a H=H a$ for all $a \in H$ it follows that every product $b a$ can be written $a b_{a}$ for some element $b_{a}$, which is unique by cancellativity. It is straightforward to check that the following identities are satisfied:

$$
e_{a}=e, \quad a_{e}=a, \quad a_{a}=a, \quad(a b)_{c}=a_{c} b_{c}, \quad\left(a_{b}\right)_{c}=a_{b c}
$$

We define a mapping $\theta$ from the opposite monoid $H^{\text {opp }}$ to the endomorphism monoid, $\operatorname{End}(H)$, of $H$ by stipulating that $\theta(a)=\theta_{a}$, where $\theta_{a}(b)=b_{a}$, for all $b \in H$. It is straightforward to show that $\theta$ is a homomorphism. Thus we may define a semidirect product on the set $H \times H^{o p p}$, by the multiplication $\langle a, b\rangle\langle c, d\rangle=$ $\left\langle a \theta_{b}(c), d b\right\rangle=\left\langle a c_{b}, d b\right\rangle$. Using the identities above, one checks that this gives an associative product, for which the element $\langle e, e\rangle$ serves as a unit element, giving us a monoid $M$.

Now we define a relation $\sim$ on $M$ by stipulating that

$$
\langle a, b\rangle \sim\langle c, d\rangle \quad \Leftrightarrow \quad a d_{b}=c b
$$

From the identities above it follows that $\sim$ defined in this manner is indeed a monoid congruence, so we may consider the algebra formed by taking the quotient by this congruence, say $G=M / \sim$. It is evident that $[\langle e, e\rangle]$ serves yet again as a unit element, and it is straightforward to check that $\langle a, b\rangle\langle b, a\rangle \sim\langle b, a\rangle\langle a, b\rangle \sim\langle e, e\rangle$, and that $\langle b, a\rangle$ is the only element (up to equivalence) such that this relation holds. Thus every element of $G$ possesses a unique inverse: $[\langle a, b\rangle]^{-1}=[\langle b, a\rangle]$, so that $G$ is a group, and a mapping defined by $a \mapsto[\langle a, e\rangle]$ yields an embedding from $H$ into $G$. That every element of $G$ is realized in the desired manner is clear.

Using this embedding result we obtain the following characterizations for the class $\mathcal{L G}^{-}$of negative cones of $\ell$-groups, which imply in particular that $\mathcal{L G}^{-}$is a variety. The equivalence of (i) and (iii) can be derived from Theorem 12 of [3], due to J. von Neumann, but we present a self-contained proof for completeness. 
Theorem 6.2. For a residuated lattice $\mathbf{L}$, the following statements are equivalent.

(i) $\mathbf{L}$ is the negative cone of an $\ell$-group.

(ii) $\mathbf{L}$ is a cancellative integral GMV-algebra.

(iii) $\mathbf{L}$ is a cancellative integral GBL-algebra.

Proof. (i) $\Rightarrow$ (ii) follows easily by direct calculation in negative cones of $\ell$-groups: for all $\mathbf{L} \in \mathcal{L G}$ and all $a, b \in L^{-}, a b / \mathbf{L}^{-} b=a b b^{-1} \wedge e=a \wedge e=a$, and $a /{ }^{\mathbf{L}^{-}}\left(b \backslash^{\mathbf{L}^{-}} a\right)=$ $a\left(b^{-1} a \wedge e\right)^{-1} \wedge e=\left(a a^{-1} b \vee a\right) \wedge e=a \vee b$.

(ii) $\Rightarrow$ (iii) is proved by Lemma 5.2 .

(iii) $\Rightarrow$ (i): We now make use of the preceding lemma to prove that any cancellative integral GBL-algebra is a member of $\mathcal{L G}^{-}$.

Let $\mathbf{L}$ be a cancellative integral GBL-algebra. If we now consider any elements $a, b \in L$, we note that $a(a \backslash b a)=b a \wedge a=b a$, since $b \leq e$ and multiplication is compatible with the lattice order. Thus there exists an element $b_{a}$, namely $a \backslash b a$, such that $b a=a b_{a}$. It follows that $L a \subseteq a L$ for all elements $a \in L$, and dually $a L \subseteq L a$. Therefore the underlying monoid of $L$ satisfies the conditions of the preceding lemma, and can be embedded into a group $G$ in the prescribed manner. Note that the group element $a b^{-1}$ corresponds to the class $[\langle a, b\rangle]$ constructed in the proof of the previous lemma. Note further that $g L=L g$ for all $g \in G$.

We consider the following standard order on $G$ : for all $a, b \in G, a \leq_{G} b$ iff $a b^{-1} \in L$. It is well known (see for example [16], p. 13) that $\leq_{G}$ is a compatible partial order on $G$ whose negative elements are precisely the elements of $L$.

We proceed to show that $\leq_{G}$ is an extension of the original order $\leq$ of $L$. More explicitly, we prove that for all $a, b \in L$,

$$
a \leq b \quad \Leftrightarrow \quad a \leq_{G} b \quad \Leftrightarrow \quad a b^{-1}=a / b \quad \Leftrightarrow \quad b^{-1} a=b \backslash a .
$$

Let $x \leq_{G} y$. Thus $x y^{-1} \in L$, and $x=x y^{-1} y \leq y$ because $x y^{-1} \leq e$. Assuming $x \leq y, x y^{-1} y=x=x \wedge y=(x / y) y$ by the generalized basic logic identity, whence cancelling $y$ gives $x y^{-1}=x / y$. Now if $x y^{-1}=x / y$, then $x=x y^{-1} y=(x / y) y=$ $x \wedge y$, hence $x \leq y$. Similarly $x \leq y$ is equivalent to $y^{-1} x=y \backslash x$. Finally if $x y^{-1}=x / y$, we have that $x y^{-1} \in L$ and thus $x \leq_{G} y$.

The preceding conclusion allows us to drop the subscript on $\leq_{G}$. We complete the proof of the theorem by showing that $\leq$ is a lattice order. Since any $\ell$-group satisfies the identity $x \vee y \approx\left(x y^{-1} \vee e\right) y$, it suffices to establish the existence of all joins of the form $g \vee e=a b^{-1} \vee e$, with $a, b \in L$.

To this end, assume that $a$ and $b$ are elements of $L$. We claim that $a b^{-1} \vee e=$ $(a \vee b) b^{-1}$. It is readily seen that $e \leq(a \vee b) b^{-1}$ and $a b^{-1} \leq(a \vee b) b^{-1}$ by multiplying these inequalities on the right by $b$.

If we consider any other element of $G$, say $c d^{-1}$ (where $c, d \in L$ ), such that both $e \leq c d^{-1}$ and $a b^{-1} \leq c d^{-1}$ hold, we have $a \leq c d^{-1} b=c d^{-1} b d d^{-1}$. Now 
we note that $b d$ and $d$ are elements of $L$ such that $b d \leq d$. Thus $(*)$ shows that $d^{-1} b d=d \backslash b d=b_{d}$. Therefore $a \leq c b_{d} d^{-1}$, so that $a d \leq c b_{d}$. Similarly, working with $e \leq c d^{-1}$, we establish $b d \leq c b_{d}$, and hence $a d \vee b d \leq c b_{d}$. Since $L$ is a residuated lattice, products distribute over joins, so we have $a \vee b \leq c b_{d} d^{-1}=c d^{-1} b$ and finally $(a \vee b) b^{-1} \leq c d^{-1}$, as desired.

We have thus succeeded in embedding a residuated lattice satisfying the identities above into a lattice-ordered group in such a way that it serves as the negative cone of the $\ell$-group.

Corollary 6.3. The variety $\mathcal{L G}^{-}$is defined, relative to $\mathcal{R L}$, by the identities $x y / y \approx x \approx y \backslash y x$ and $(x / y) y \approx x \wedge y \approx y(y \backslash x)$. Alternatively, the last two identities can be replaced by $x /(y \backslash x) \approx x \vee y \approx(x / y) \backslash x$.

\section{The subvarieties of $\mathcal{L G}$ and $\mathcal{L G}^{-}$}

We now extend the map ${ }^{-}: \mathcal{L G} \rightarrow \mathcal{L G}^{-}$to subclasses of $\mathcal{L G}$, and in particular to the lattice of subvarieties $\mathbf{L}(\mathcal{L G})$. We show that the image of a variety is always a variety, that every subvariety of $\mathcal{L G}^{-}$is obtained in this way and that the map is an order isomorphism, hence a lattice isomorphism (see Figure 4).

In the second part of this section we show how equational bases can be translated back and forth between corresponding varieties of $\mathcal{L G}$ and $\mathcal{L G}^{-}$. We conclude the section by indicating how these results are related to R. McKenzie's general characterization of categorical equivalence [22].

Recall that for a class $\mathcal{K}$ of residuated lattices, $\mathbf{H}(\mathcal{K}), \mathbf{S}(\mathcal{K}), \mathbf{P}(\mathcal{K})$ and $\mathcal{K}^{-}$denote, respectively, the class of homomorphic images, subalgebras, products and negative cones of members of $\mathcal{K}$.

Theorem 7.1. The map $\mathcal{K} \mapsto \mathcal{K}^{-}$, defined on classes of $\ell$-groups, commutes with the operators $\mathbf{H}, \mathbf{S}$ and $\mathbf{P}$, and restricts to a lattice isomorphism between the subvariety lattices of $\mathcal{L G}$ and $\mathcal{L G}^{-}$.

Proof. Consider any subclass $\mathcal{K}$ of $\mathcal{L G}$. To see that $\mathbf{S}\left(\mathcal{K}^{-}\right)=(\mathbf{S} \mathcal{K})^{-}$, let $\mathbf{G}^{-}$be a member of $\mathcal{K}^{-}$and let $\mathbf{H}^{\prime}$ be a subalgebra of $\mathbf{G}^{-}$. By the definition of $\mathcal{K}^{-}$, there exists $\mathbf{G} \in \mathcal{K}$ whose negative cone is $\mathbf{G}^{-}$. We show that $\mathbf{H}^{\prime}$ is the negative cone of a subalgebra $\mathbf{H}$ of $\mathbf{G}$.

Consider the set $H=\left\{a b^{-1}: a, b \in H^{\prime}\right\}$. We establish that $H$ is the subgroup of $\mathbf{G}$ generated by $H^{\prime}$ by proving that $H$ is closed under products in $\mathbf{G}$. Let $a b^{-1}$ and $c d^{-1}$ be in $H$. Invoking the proof of Theorem 6.2 (iii) $\Rightarrow$ (i), we have

$$
a b^{-1} c d^{-1}=a b^{-1} c b b^{-1} d^{-1}=a(b \backslash c b)(d b)^{-1}=a c_{b}(d b)^{-1},
$$

which is again an element of $H$ since $\mathbf{H}^{\prime}$ is a subalgebra of $\mathbf{G}^{-}$. What we know so far is that $H$ is a partially ordered group with respect to the order of $\mathbf{G}$. To show 
that $H$ is a subalgebra ( $\ell$-subgroup) of $\mathbf{G}$, it will suffice to show that for $a, b \in H^{\prime}$ the join $a b^{-1} \vee e$ (in $\mathbf{G}$ ) is an element of $H$. Let $a, b \in H^{\prime}$, and recall from the proof of Theorem 6.2 that $a b^{-1} \vee^{\mathbf{G}} e=\left(a \vee^{\mathbf{G}^{-}} b\right) b^{-1}$. Since $\mathbf{H}^{\prime}$ is a subalgebra of $\mathbf{G}^{-}$, we have $a \vee^{\mathbf{G}^{-}} b=a \vee^{\mathbf{H}^{\prime}} b$. It follows that $a b^{-1} \vee^{\mathbf{G}} e \in H$, as was to be shown.

Since $\mathbf{G}$ is an algebra in $\mathcal{K}$, with subalgebra $\mathbf{H}$, this establishes that $\mathbf{S}\left(\mathcal{K}^{-}\right) \subseteq$ $(\mathbf{S} \mathcal{K})^{-}$. The reverse inclusion is immediate.

The fact that $\mathbf{P}\left(\mathcal{K}^{-}\right)=(\mathbf{P} \mathcal{K})^{-}$follows from the observation that for a collection of residuated lattices $\left\{\mathbf{G}_{i}\right\}_{i \in I}$ in $\mathcal{K}$,

$$
\prod_{i \in I} \mathbf{G}_{i}^{-}=\left(\prod_{i \in I} \mathbf{G}_{i}\right)^{-} .
$$

Finally we must show that $\mathbf{H}\left(\mathcal{K}^{-}\right)=(\mathbf{H} \mathcal{K})^{-}$. So let $\mathbf{G}^{-} \in \mathcal{K}^{-}$and consider a surjective residuated lattice homomorphism $\phi: \mathbf{G}^{-} \rightarrow \mathbf{H}^{\prime}$. As suggested by the notation, $\mathbf{G}^{-}$is the negative cone of an $\ell$-group $\mathbf{G} \in \mathcal{K}$, and since $\mathcal{L G}^{-}$is a variety, we may assume that $\mathbf{H}^{\prime}$ is the negative cone of an $\ell$-group $\mathbf{H}$. We extend $\phi$ to $\bar{\phi}: \mathbf{G} \rightarrow \mathbf{H}$ by defining $\bar{\phi}\left(a b^{-1}\right)=\phi(a) \phi(b)^{-1}$. We need to show that this map is a well-defined $\ell$-group homomorphism onto $\mathbf{H}$.

To show that $\bar{\phi}$ is well-defined, consider two different representations for the same element of $\mathbf{G}: a b^{-1}=c d^{-1}$. This is the case if and only if $a d_{b}=c b$, implying that $\phi(a) \phi(d)_{\phi(b)}=\phi(c) \phi(b)$ since $\phi$ is a homomorphism. This in turn implies that $\phi(a) \phi(b)^{-1}=\phi(c) \phi(d)^{-1}$.

The map $\bar{\phi}$ is clearly onto, since $\phi$ is onto and every element $x \in H$ can be written as $a b^{-1}$ for some $a$ and $b$ in $H^{\prime}$.

To show that $\bar{\phi}$ is a group homomorphism, let $a b^{-1}$ and $c d^{-1}$ be elements of G. Recall that $a b^{-1} c d^{-1}=a b^{-1} c b b^{-1} d^{-1}=a c_{b}(d b)^{-1}$ so that $\bar{\phi}\left(a b^{-1} c d^{-1}\right)=$ $\bar{\phi}\left(a c_{b}(d b)^{-1}\right)=\phi\left(a c_{b}\right) \phi(d b)^{-1}=\phi(a) \phi(c)_{\phi(b)} \phi(b)^{-1} \phi(d)^{-1}$, since $\phi$ is a homomorphism. But this gives

$$
\phi(a) \phi(b)^{-1} \phi(c) \phi(b) \phi(b)^{-1} \phi(d)^{-1}=\bar{\phi}\left(a b^{-1}\right) \bar{\phi}\left(c d^{-1}\right) .
$$

Finally, we show that $\bar{\phi}$ preserves joins. As before, it suffices to consider joins of the form $a b^{-1} \vee e$. Recalling that $a b^{-1} \vee e=(a \vee b) b^{-1}$, we have

$$
\bar{\phi}\left(a b^{-1} \vee e\right)=\bar{\phi}\left((a \vee b) b^{-1}\right)=\phi(a \vee b) \phi(b)^{-1}=(\phi(a) \vee \phi(b)) \phi(b)^{-1},
$$

since $\phi$ preserves joins. But this last product is $\phi(a) \phi(b)^{-1} \vee e=\bar{\phi}\left(a b^{-1}\right) \vee e$. Thus $\bar{\phi}$ is a lattice homomorphism as well.

This shows that $\mathbf{H} \in \mathbf{H} \mathcal{K}$, hence $\mathbf{H}^{\prime}=\mathbf{H}^{-} \in(\mathbf{H} \mathcal{K})^{-}$, as desired. Again the reverse inclusion is straightforward.

The preceding considerations immediately imply that if $\mathcal{V}$ is a variety of $\ell$ groups, then $\mathcal{V}^{-}$is a subvariety of $\mathcal{L G}^{-}$. Moreover, the map $\mathcal{V} \mapsto \mathcal{V}^{-}$is clearly order preserving. To show it is onto, let $\mathcal{W} \in \mathbf{L}\left(\mathcal{L G}^{-}\right)$and consider the class 


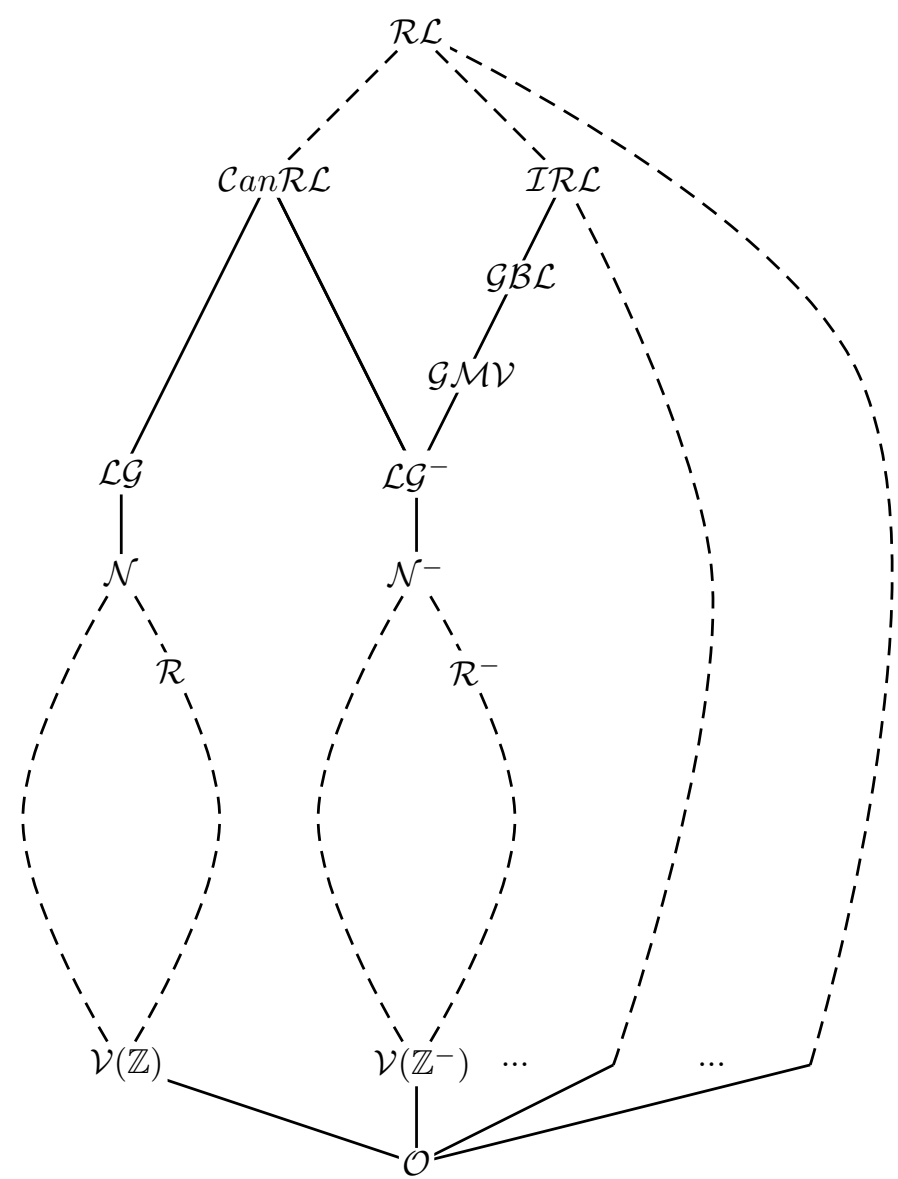

FiguRE 4. Inclusions between some subvarieties of $\mathcal{R} \mathcal{L}$

$\mathcal{K}=\left\{\mathbf{L} \in \mathcal{L G}: \mathbf{L}^{-} \in \mathcal{W}\right\}$. Then $\mathcal{K}^{-}=\mathcal{W}$ and $\mathcal{K}$ is a variety since $\mathbf{L} \in \mathbf{H S P} \mathcal{K}$ implies $\mathbf{L}^{-} \in(\mathbf{H S P} \mathcal{K})^{-}=\mathbf{H S P}\left(\mathcal{K}^{-}\right)=\mathcal{W}$, so $\mathbf{L} \in \mathcal{K}$.

Finally, we show that the map is one-to-one and reflects the order as well. Indeed, an $\ell$-group is determined up to isomorphism by its negative cone (the preceding map $\bar{\phi}$ is an isomorphism when $\phi$ is an isomorphism), so if $\mathcal{V} \not \leq \mathcal{V}^{\prime}$ and $G \in \mathcal{V}-\mathcal{V}^{\prime}$, then $G^{-} \in \mathcal{V}^{-}-\mathcal{V}^{-}$. It follows that $\mathcal{V} \mapsto \mathcal{V}^{-}$is a lattice isomorphism between the two subvariety lattices.

Corollary 7.2. The variety $\mathcal{V}\left(\mathbb{Z}^{-}\right)$consists of all negative cones of abelian $\ell-$ groups. 
It was proved above that there is a one-to-one correspondence between subvarieties of $\mathcal{L G}$ and $\mathcal{L G}^{-}$. Since the proof made use of the HSP characterization of varieties, it gave no insight into how one might find an equational basis for $\mathcal{V}^{-}$ given a basis for $\mathcal{V}$, and vice versa. We proceed to do that in the remainder of this section.

From subvarieties of $\mathcal{L G}^{-}$to subvarieties of $\mathcal{L G}$. In this direction, the translation is derived essentially from the definition of the negative cone. For a residuated lattice term $t$, we define a translated term $t^{-}$by

$$
\begin{gathered}
x^{-}=x \wedge e \quad e^{-}=e \\
(s / t)^{-}=s^{-} / t^{-} \wedge e \quad(s \backslash t)^{-}=s^{-} \backslash t^{-} \wedge e \\
(s t)^{-}=s^{-} t^{-} \quad(s \vee t)^{-}=s^{-} \vee t^{-} \quad(s \wedge t)^{-}=s^{-} \wedge t^{-}
\end{gathered}
$$

Lemma 7.3. Let $\mathbf{L} \in \mathcal{R} \mathcal{L}$ and consider any $\mathcal{R} \mathcal{L}$ term $t$. For any $a_{1}, \ldots, a_{n} \in L$,

$$
t^{-\mathbf{L}}\left(a_{1}, \ldots, a_{n}\right)=t^{\mathbf{L}^{-}}\left(a_{1} \wedge e, \ldots, a_{n} \wedge e\right)
$$

Proof. By definition this is true for variables and the constant term $e$. Assume the statement holds for terms $s$ and $t$. Then

$$
\begin{aligned}
(s / t)^{-\mathbf{L}}\left(a_{1}, \ldots, a_{n}\right) & =\left(s^{-\mathbf{L}}\left(a_{1}, \ldots, a_{n}\right) / \mathbf{L}^{-\mathbf{L}}\left(a_{1}, \ldots, a_{n}\right)\right) \wedge e \\
& =\left(s^{\mathbf{L}^{-}}\left(a_{1} \wedge e, \ldots, a_{n} \wedge e\right) /{ }^{\mathbf{L}} t^{\mathbf{L}^{-}}\left(a_{1} \wedge e, \ldots, a_{n} \wedge e\right)\right) \wedge e \\
& =(s / t)^{\mathbf{L}^{-}}\left(a_{1} \wedge e, \ldots, a_{n} \wedge e\right)
\end{aligned}
$$

and similar inductive steps for $\backslash, \cdot, \vee, \wedge$ complete the proof.

Lemma 7.4. For any $\mathbf{L} \in \mathcal{R} \mathcal{L}, \mathbf{L}^{-} \models s \approx t$ iff $\mathbf{L} \models s^{-} \approx t^{-}$.

Proof. Suppose $\mathbf{L}^{-} \models s \approx t$, and let $a_{1}, \ldots, a_{n} \in L$. By the preceding lemma, $s^{-\mathbf{L}}\left(a_{1}, \ldots, a_{n}\right)=s^{\mathbf{L}^{-}}\left(a_{1} \wedge e, \ldots, a_{n} \wedge e\right)=t^{\mathbf{L}^{-}}\left(a_{1} \wedge e, \ldots, a_{n} \wedge e\right)=t^{-\mathbf{L}}\left(a_{1}, \ldots, a_{n}\right)$, hence $\mathbf{L} \models s^{-} \approx t^{-}$. The reverse implication is similar and uses the observation that for $a_{i} \in L^{-}, a_{i}=a_{i} \wedge e$.

Theorem 7.5. Let $\mathcal{V}$ be a subvariety of $\mathcal{L G}^{-}$, defined by a set $\mathcal{E}$ of identities and let $\mathcal{W}=\operatorname{Mod}\left(\mathcal{E}^{-}\right) \cap \mathcal{L} \mathcal{G}$, where $\mathcal{E}^{-}=\left\{s^{-} \approx t^{-}:(s \approx t) \in \mathcal{E}\right\}$. Then $\mathcal{W}^{-}=\mathcal{V}$.

Proof. Consider $\mathbf{M} \in \mathcal{W}^{-}$, which means there exists an $\mathbf{L} \in \mathcal{W}$ such that $\mathbf{M}$ is isomorphic to $\mathbf{L}^{-}$. Then $\mathbf{L} \models \mathcal{E}^{-}$, and by the previous lemma this is equivalent to $\mathbf{L}^{-} \models \mathcal{E}$, which in turn is equivalent to $\mathbf{L}^{-} \in \mathcal{V}$. Hence $\mathbf{M} \in \mathcal{V}$.

Conversely, let $\mathbf{M} \in \mathcal{V}$. Then there exists an $\ell$-group $\mathbf{L}$ such that $\mathbf{M}$ is isomorphic to $\mathbf{L}^{-}$. ( $\mathbf{L}$ is constructed as in Theorem 6.2.) Using the previous lemma again, we get that $\mathbf{L}=\mathcal{E}^{-}$, hence $\mathbf{M} \in \mathcal{W}^{-}$. 
As an example, consider the variety $\mathcal{N}^{-}$that is defined by the identity $x^{2} y^{2} \leq y x$ relative to $\mathcal{L G}^{-}$. The corresponding identity for the variety $\mathcal{N}$ of normal valued $\ell$-groups is $(x \wedge e)^{2}(y \wedge e)^{2} \leq(y \wedge e)(x \wedge e)$.

From subvarieties of $\mathcal{L G}$ to subvarieties of $\mathcal{L G}^{-}$. Note that since $\cdot$ and ${ }^{-1}$ distribute over $\vee$ and $\wedge$, any $\mathcal{L G}$ identity is equivalent to a conjunction of two identities of the form $e \leq p\left(g_{1}, \ldots, g_{n}\right)$, where $p$ is a lattice term and $g_{1}, \ldots, g_{n}$ are group terms. Since $\ell$-groups are distributive, this can be further reduced to a finite conjunction of inequalities of the form $e \leq g_{1} \vee \cdots \vee g_{n}$.

For a term $t\left(x_{1}, \ldots, x_{m}\right)$ and a variable $z$ distinct from $x_{1}, \ldots, x_{m}$, let

$$
\bar{t}\left(z, x_{1}, \ldots, x_{m}\right)=t\left(z^{-1} x_{1}, \ldots, z^{-1} x_{m}\right) .
$$

Lemma 7.6. Let $\mathbf{L}$ be an $\ell$-group, and $t$ an $\ell$-group term. Then

$$
\begin{gathered}
\mathbf{L} \models e \leq t\left(x_{1}, \ldots, x_{m}\right) \quad \text { iff } \\
\mathbf{L} \models x_{1} \vee \cdots \vee x_{m} \vee z \leq e \Rightarrow e \leq \bar{t}\left(z, x_{1}, \ldots, x_{m}\right) .
\end{gathered}
$$

Proof. In the forward direction this is obvious. To prove the reverse implication, assume the right hand side holds and let $a_{1}, \ldots, a_{m} \in L$. Define $c=a_{1}^{-1} \wedge \cdots \wedge a_{m}^{-1} \wedge e$ and $b_{i}=c a_{i}$ for $i=1, \ldots, m$. Then $c \leq e$ and $c \leq a_{i}^{-1}$, hence $b_{i} \leq e$. Now by assumption, $e \leq t\left(c^{-1} b_{1}, \ldots, c^{-1} b_{m}\right)=t\left(a_{1}, \ldots, a_{m}\right)$.

Lemma 7.7. Let $\mathbf{L} \in \mathcal{L G}$. For any group term g, there exist an $\mathcal{R} \mathcal{L}$ term $\hat{g}$ such that $\left.(g \wedge e)^{\mathbf{L}}\right|_{L^{-}}=\hat{g}^{\mathbf{L}^{-}}$.

Proof. Essentially we have to rewrite group terms so that all the variables with inverses appear at the beginning of the term. This is done using conjugation: $x y^{-1}=y^{-1}\left(y x y^{-1}\right)=y^{-1}(y x / y)$. Note that $L^{-}$is closed under conjugation by arbitrary elements, since $x \leq e$ implies $y x y^{-1} \leq e$. If we also have $y \leq e$, then $y x \in L^{-}$and $y x \leq y$, hence $y x / \mathbf{L}^{-} y=y x /{ }^{\mathbf{L}} y$.

To describe the translation of an arbitrary group term, we may assume that it is of the form $p_{1} q_{1}^{-1} p_{2} q_{2}^{-1} \cdots p_{n} q_{n}^{-1}$ where the $p_{i}$ and $q_{i}$ are products of variables (without inverses). By using conjugation, we write this term in the form

$$
q_{1}^{-1} q_{2}^{-1} \cdots q_{n}^{-1}\left(q_{n}\left(\cdots\left(q_{2}\left(q_{1} p_{1} / q_{1}\right) p_{2} / q_{2}\right) \cdots\right) p_{n} / q_{n}\right) .
$$

So we can take $\hat{g}=s \backslash t$ where

$$
s=q_{n} \cdots q_{2} q_{1} \text { and } t=q_{n}\left(\cdots\left(q_{2}\left(q_{1} p_{1} / q_{1}\right) p_{2} / q_{2}\right) \cdots\right) p_{n} / q_{n} .
$$

Corollary 7.8. Let $g_{1}, \ldots, g_{n}$ be group terms with variables among $x_{1}, \ldots, x_{m}$. For any $\ell$-group $\mathbf{L}$,

$$
\mathbf{L}^{-} \models \hat{g}_{1} \vee \cdots \vee \hat{g}_{n} \approx e \text { iff } \mathbf{L} \models x_{1} \vee \cdots \vee x_{m} \leq e \Rightarrow e \leq g_{1} \vee \cdots \vee g_{n} .
$$


For the next result, recall the discussion about identities in $\ell$-groups, and the definition of $\bar{t}$ at the beginning of this subsection.

Theorem 7.9. Let $\mathcal{V}$ be a subvariety of $\mathcal{L} \mathcal{G}$, defined by a set $\mathcal{E}$ of identities, which we may assume are of the form $e \leq g_{1} \vee \cdots \vee g_{n}$. Let

$$
\overline{\mathcal{E}}=\left\{e \approx \widehat{\overline{g_{1}}} \vee \cdots \vee \widehat{\overline{g_{n}}}: e \leq g_{1} \vee \cdots \vee g_{n} \text { is in } \mathcal{E}\right\}
$$

Then $\overline{\mathcal{E}}$ is an equational basis for $\mathcal{V}^{-}$relative to $\mathcal{L G}^{-}$.

Proof. By construction, any member of $\mathcal{V}^{-}$satisfies all the identities in $\overline{\mathcal{E}}$. On the other hand, if $\mathbf{M} \in \mathcal{L G}^{-}$is a model of the identities in $\overline{\mathcal{E}}$, then $\mathbf{M}$ is the negative cone of some $\mathbf{L} \in \mathcal{L} \mathcal{G}$. From the reverse directions of Corollary 7.8 and Lemma 7.6 we infer that $\mathbf{L}$ satisfies the equations in $\mathcal{E}$, hence $\mathbf{M} \in \mathcal{V}^{-}$.

For example consider the variety $\mathcal{R}$ of representable $\ell$-groups which (by definition) is generated by the class of totally ordered groups (see [1] for more details). An equational basis for this variety is given by $e \leq x^{-1} y x \vee y^{-1}$ (relative to $\mathcal{L} \mathcal{G}$ ). Applying the translation above, we obtain $e \approx z x \backslash(z y / z) x \vee y \backslash z$ as as equational basis for $\mathcal{R}^{-}$.

Corollary 7.10. The map $\mathcal{V} \mapsto \mathcal{V}^{-}$from $\mathbf{L}(\mathcal{L G})$ to $\mathbf{L}\left(\mathcal{L G}^{-}\right)$sends finitely based subvarieties of $\mathcal{L G}$ to finitely based subvarieties of $\mathcal{L G}^{-}$.

Categorical equivalence and the functor $\mathbf{L} \mapsto \mathbf{L}^{-}$. The connection between $\mathcal{L G}$ and $\mathcal{L G}^{-}$is actually a special case of a categorical equivalence. In the algebraic setting such equivalences were characterized by R. McKenzie in [22] by combinations of the following two constructions.

Let $\mathbf{A}$ be an algebra, and let $T$ be the set of all terms in the language of $\mathbf{A}$. Given a unary term $\sigma$ we define a new algebra called the $\sigma$-image of $\mathbf{A}$ by $\mathbf{A}(\sigma)=$ $\left\langle\sigma(A),\left\{t_{\sigma}: t \in T\right\}\right\rangle$, where $t_{\sigma}^{\mathbf{A}(\sigma)}\left(x_{1}, \ldots, x_{n}\right)=\sigma^{\mathbf{A}}\left(t^{\mathbf{A}}\left(x_{1}, \ldots, x_{n}\right)\right)$.

The second construction is the matrix power of $\mathbf{A}$. Let $T_{k}$ be the set of $k$-ary terms. For a positive integer $n$ we define

$$
\mathbf{A}^{[n]}=\left\langle A^{n},\left\{m_{t}: t \in\left(T_{k n}\right)^{n} \text { for some } k>0\right\}\right\rangle,
$$

where $m_{t}:\left(A^{n}\right)^{k} \rightarrow A^{n}$ is given by $m_{t}\left(\bar{x}_{1}, \ldots, \bar{x}_{k}\right)_{i}=t_{i}^{\mathbf{A}}\left(x_{11}, \ldots, x_{k n}\right)$.

For a class $\mathcal{K}$ of algebras we let $\mathcal{K}(\sigma)$ and $\mathcal{K}^{[n]}$ be the classes of $\sigma$-images and $n$-th matrix powers respectively. A term $\sigma$ is idempotent in $\mathcal{K}$ if $\mathcal{K} \models \sigma(\sigma(x))=\sigma(x)$, and it is invertible in $\mathcal{K}$ if there exist unary terms $t_{1}, \ldots, t_{n}$ and an $n$-ary term $t$ (for some $n>0$ ) such that

$$
\mathcal{K} \models x \approx t\left(\sigma\left(t_{1}(x)\right), \ldots, \sigma\left(t_{n}(x)\right)\right) .
$$

A central result in [22] is the following. 
Theorem 7.11. Two varieties $\mathcal{V}$ and $\mathcal{W}$ are categorically equivalent if and only if there is an $n>0$ and an invertible idempotent term $\sigma$ for $\mathcal{V}^{[n]}$ such that $\mathcal{W}$ is term-equivalent to $\mathcal{V}^{[n]}(\sigma)$.

In the setting of $\ell$-groups and their negative cones, we can see an instance of this result. The term $\sigma(x)=x \wedge e$ is certainly idempotent, and it is invertible (with $n=2)$ since $x \approx(x \wedge e)\left(x^{-1} \wedge e\right)^{-1}$ holds in all $\ell$-groups. Of course $\mathbf{L}(\sigma)$ is not of the same type as $\mathbf{L}^{-}$, but with the help of Lemma 7.7 it is easy to see that they are term equivalent. In the other direction, every member of $\mathcal{L G}^{-}$can be mapped to a $\tau$-image of a matrix square that is term-equivalent to an $\ell$-group. In general, the term $\tau$ is given by $\tau(\bar{x})=\left\langle\sigma t_{1} t(\bar{x}), \ldots, \sigma t_{n} t(\bar{x})\right\rangle$, which reduces to $\tau(\langle x, y\rangle)=\langle x / y, y / x\rangle$ for negative cones.

\section{Open problems}

Problem 8.1. Is $\mathcal{C}$ an $\mathcal{R} \mathcal{L}$ join-irreducible or completely join-irreducible in $\mathbf{L}(\mathcal{R} \mathcal{L})$ ?

Problem 8.2. Is every lattice a subreduct of a commutative cancellative residuated lattice (see Theorem 4.3)?

Problem 8.3. Are there commutative, cancellative, distributive residuated lattices that are not in $\mathcal{C}$ an $\mathcal{R} \mathcal{L}^{C}$ ? In the noncommutative case the 2 -generated free $\ell$-group is an example.

Problem 8.4. Characterize the class of monoids that are monoid reducts of $\mathcal{C}$ an $\mathcal{R} \mathcal{L}$.

Problem 8.5. Do $\mathcal{C} a n \mathcal{R} \mathcal{L}$ and $\mathcal{C}$ an $\mathcal{R} \mathcal{L}^{C}$ have decidable equational theories?

Problem 8.6. Is there a Weinberg-type description of free algebras in $\mathcal{C}$ an $\mathcal{R} \mathcal{L}^{C}$ ? See e.g. Powell and Tsinakis [25].

\section{REFERENCES}

[1] M. Anderson and T. Feil, Lattice-Ordered Groups: an introduction, D. Reidel Publishing Company, 1988.

[2] J. Berman, Homogeneous lattices and lattice-ordered groups, Colloq. Math. 32 (1974), 13-24.

[3] G. Birkhoff, Lattice-ordered groups, Ann. Math. 43 (1942), 298-331.

[4] G. Birkhoff, Lattice Theory (3rd ed), Coloquium Publications 25, Amer. MAth. Soc., 1967.

[5] W. J. Blok and I. M. A. Ferreirim, On the structure of hoops, Algebra Universalis [43] (2000), 233-257.

[6] W. J. Blok and J. G. Raftery, Varieties of commutative residuated integral pomonoids and their residuation subreducts, J. Algebra 190 (1997), 280-328.

[7] K. Blount and C. Tsinakis, The structure of Residuated Lattices, Internat. J. Algebra Comput., to appear.

[8] T. S. Blyth and M. F. Janowitz, Residuation Theory, Pergamon Press, 1972.

[9] B. Bosbach, Komplementäre Halbgruppen: Axiomatik und Arithmetik. (German) Fund. Math. 64 (1969), 257-287. 
[10] B. Bosbach, Komplementäre Halbgruppen: Kongruenzen und Quotienten. (German) Fund. Math. 69 (1970), 1-14.

[11] C.-C. Chang, Algebraic analysis of many valued logics, Trans. Amer. Math. Soc. 88 (1958) 467-490.

[12] C.-C. Chang, A new proof of the completeness of the Eukasiewicz axioms, Trans. Amer. Math. Soc. 93 (1959) 74-80.

[13] R. Cignoli, I. D’Ottaviano, D. Mundici, Algebraic foundations of many-valued reasoning, Trends in Logic - Studia Logica Library, 7. Kluwer Academic Publishers, Dordrecht, 2000.

[14] J. Cole, Residuated Lattice Orderings on Free Monoids, Qualifying paper, Vanderbilt, 2000.

[15] I. M. A. Ferreirim, On varieties and quasivarieties of hoops and their reducts, Ph.D. thesis, University of Illinois at Chicago, 1992.

[16] L. Fuchs, Partially ordered algebraic systems, Pergamon Press, 1963.

[17] N. Galatos, Minimal varieties of residuated lattices, preprint.

[18] N. Galatos and C. Tsinakis Generalized $M V$-algebras, in preparation.

[19] P. Hájek, Metamathematics of Fuzzy Logic, volume 4 of Trends in Logic, Kluwer Academic Publishers, 1998.

[20] J. Hart, L. Rafter and C. Tsinakis, The structure of Commutative Residuated lattices, Internat. J. Algebra Comput. 12 (2002), 509-524.

[21] P. Jipsen and C. Tsinakis, A survey of Residuated Lattices, Ordered Algebraic Structures (J. Martinez, editor), Kluwer Academic Publishers, Dordrecht, 2002, pp. 19-56.

[22] R. McKenzie, An algebraic version of categorical equivalence for varieties and more general algebraic categories, Logic and Algebra (ed. A. Ursini, P. Aglianò), Marcel Dekker, 1996, pp. $211-244$.

[23] D. Mundici, Interpretation of $A F C^{*}$-algebras in Eukasiewicz sentential calculus, J. Funct. Anal. 65 (1986), 15-63.

[24] V. Novák, I. Perfilieva, and J. Mockor, Mathematical Principles of Fuzzy Logic, volume 517 of The Kluwer International Series in Engineering and Computer Science, Kluwer Academic Publishers, 1999.

[25] W. B. Powell and C. Tsinakis, Free products in varieties of lattice-ordered groups, in Lattice-Ordered Groups (A. M. W. Glass and W. C. Holland, Editors), Kluwer Academic Publishers, 1989, pp. 278-307.

[26] M. Ward, R.P. Dilworth, Residuated Lattices, Transactions of the AMS 45 (1939), 335-354.

[27] E. C. Weinberg, Free lattice-ordered abelian groups, Math. Ann. 151 (1963), 187-199.

P. Bahls, J. Cole, N. Galatos, P. Jipsen, and C. Tsinakis

Department of Mathematics, Vanderbilt University, 1326 Stevenson Center, Nashville, TN 37240, USA

e-mail: pbahls@math.uiuc.edu

e-mail: jcole@huntingdon.edu

e-mail: galatos@jaist.ac.jp

e-mail: jipsen@chapman.edu

e-mail: Constantine.Tsinakis@vanderbilt.edu

(12) To access this journal online:

